\title{
Biocatalytic oxidative cross-coupling reactions for biaryl bond formation
}

\author{
Lara E. Zetzsche\#, Jessica A. Yazarians", Suman Chakrabarty, Meagan E. Hinze, Lauren A. M. Murray, \\ April L. Lukowski, Leo A. Joyce, and Alison R. H. Narayan*
}

Summary | Despite their varied purposes, many indispensable molecules in medicine, materials, and asymmetric catalysis share a biaryl core. The necessity of joining arene building blocks to access these valuable compounds has inspired multiple approaches for biaryl bond formation and challenged chemists to develop increasingly concise and robust methods for this task. Oxidative coupling of two $\mathrm{C}-\mathrm{H}$ bonds offers an efficient strategy for the formation of a biaryl $\mathrm{C}-\mathrm{C}$ bond, however, fundamental challenges remain in controlling the reactivity and selectivity for uniting a given pair of substrates. Biocatalytic oxidative cross-coupling reactions have the potential to overcome limitations inherent to small moleculemediated methods by providing a paradigm with catalyst-controlled selectivity. In this article, we disclose a strategy for biocatalytic cross-coupling through oxidative $\mathrm{C}-\mathrm{C}$ bond formation using cytochrome $\mathrm{P} 450$ enzymes. We demonstrate the ability to catalyze cross-coupling reactions on a panel of phenolic substrates using natural P450 catalysts. Moreover, we engineer a P450 to possess the desired reactivity, siteselectivity, and atroposelectivity by transforming a low-yielding, unselective reaction into a highly efficient and selective process. This streamlined method for constructing sterically hindered biaryl bonds provides a programmable platform for assembling molecules with catalyst-controlled reactivity and selectivity.

Biaryl bonds are ubiquitous in drugs, materials, and ligands for asymmetric catalysis (Figure 1a). ${ }^{1,2}$ The most commonly employed strategy to construct these molecules relies on metal-catalyzed cross-coupling of prefunctionalized aryl fragments, thereby of fering preprogrammed site-selectivity, albeit at the expense of adding extra steps in a synthetic campaign (Figure 1b). ${ }^{3-5}$ These reactions can require extensive screening to identify suitable conditions for each new pair of coupling partners and are still restricted in the connectivities which are readily accessible, ${ }^{6-10}$ with the formation of tetra-ortho-substituted biaryl bonds remaining an outstanding challenge. ${ }^{11,12}$ Oxidative coupling provides an alternative strategy for biaryl bond formation through the net transformation of two $\mathrm{C}-\mathrm{H}$ bonds into a $\mathrm{C}-\mathrm{C}$ bond (Figure $1 \mathrm{~b}$ ). ${ }^{13,14}$ However, these advantages come at the expense of the efficiency and selectivity of this transformation, which is typically dictated by the intrinsic stereoelectronic properties of each substrate, thereby limiting the versatility of this transformation (Figure 1c). ${ }^{14-17}$ Numerous metal catalysts and metal-free oxidants have been reported for the dimerization and cross-coupling of phenolic substrates; however, the application of these methods is commonly restricted to electron-rich phenols while oxidative coupling with electron deficient phenols remains more broadly challenging. ${ }^{15-26}$ When general reactivity can be achieved, controlling the chemo-, site-, and atroposelectivity of the oxidative coupling presents a considerable hurdle, particularly in cases where the intrinsic steric and electronic properties of the reacting phenols prohibit access to the desired product isomer. ${ }^{16,17,27-31}$

Biocatalytic oxidative cross-coupling reactions have the potential to overcome the limitations inherent to small molecule-mediated methods for direct oxidative coupling by providing a paradigm with catalyst-controlled selectivity. Nature hasevolved oxidative enzymes, including laccases and cytochromes P450 (P450s), that mediate the dimerization of phenolic compounds in the biosynthesis of biaryl natural products (Supplemental Table S2) ${ }^{32}$ The application of laccases in selective catalysis has been limited by the scarcity of examples in which these enzymes exert control over the selectivity of the bond formation following the initial oxidation..$^{33-35}$ In contrast, an ever-expanding repertoire of P450s mediate highly selective oxidation reactions ${ }^{36}$ among which exist a small subset of enzymes that catalyze site- and 


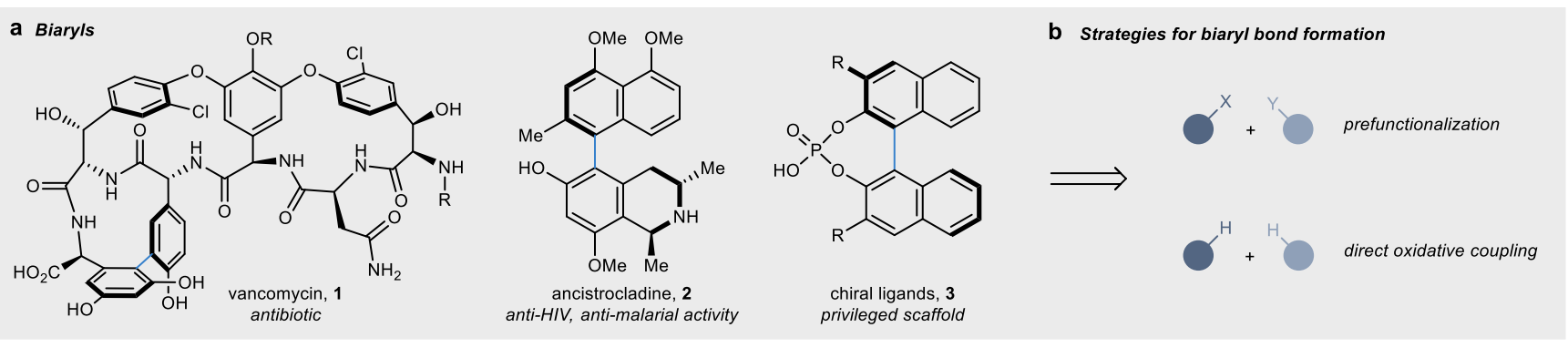

C Challenges in the direct oxidative coupling of phenols
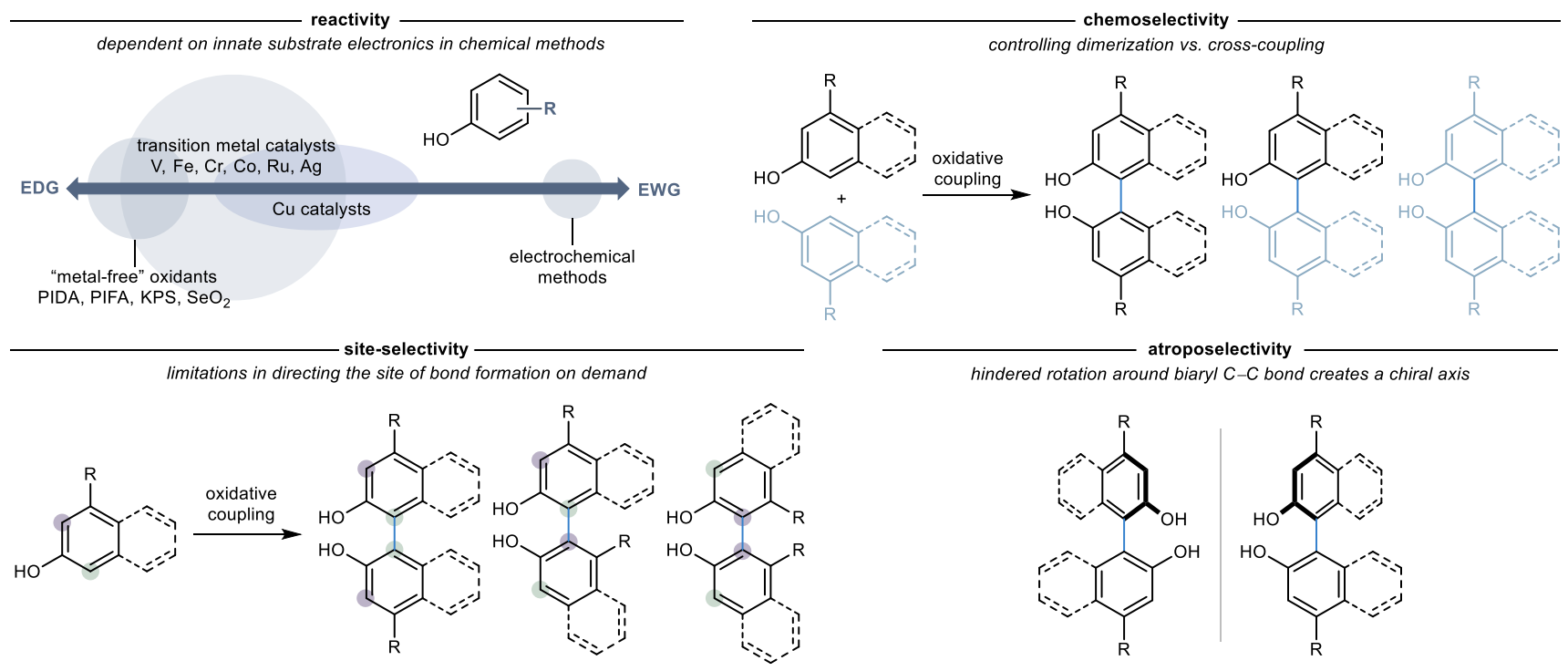

hindered rotation around biaryl C-C bond creates a chiral axis

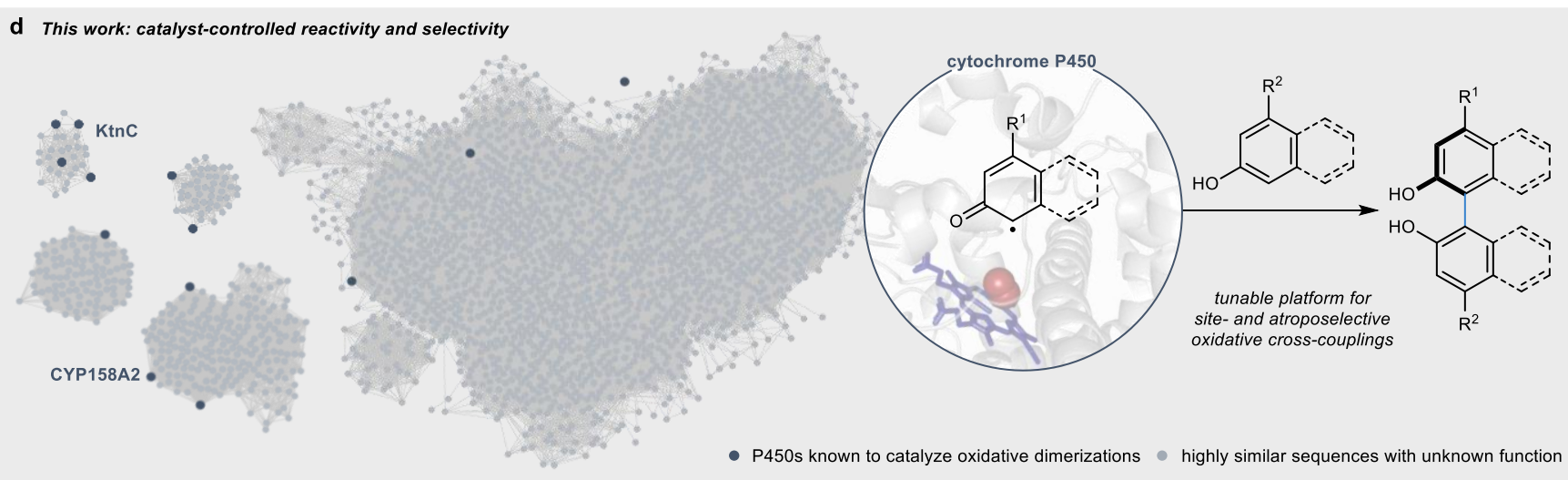

Fig. 1 | Biaryl bond formation through direct oxidative cross-coupling. a, The biaryl scaffold is a privileged substructure in drug discovery and asymmetric catalysis. b, Biaryl bonds can be accessed through metal-catalyzed cross-coupling of prefunctionalized starting materials or direct oxidative coupling. c, Oxidative biaryl bond formation faces fundamental challenges in reactivity and selectivity. $\mathbf{d}$, In this work, we report the first biocatalytic platform for the selective cross-coupling of phenolic substrates through oxidative $\mathrm{C}-\mathrm{C}$ bond formation.

atroposelective dimerizations. ${ }^{37}$ This subset of wild-type P450s that perform oxidative coupling chemistry are yet to be explored as biocatalysts for unnatural cross-coupling reactions. Moreover, these catalysts exist within a large pool of related enzyme sequences with untapped potential to catalyze a diverse array of oxidative coupling reactions (see sequence similarity network, SSN, in Figure 1d). We hypothesized that this class of enzymes could be developed into tunable biocatalysts for convergent oxidative crosscoupling reactions with catalyst-controlled reactivity, site-selectivity, and atroposelectivity.

Among the P450s previously identified as catalysts for oxidative dimerization en route to biaryl natural products (Supplemental Table S2), we were initially drawn to a small cluster of Aspergillus 
enzymes that each impart unique site-selectivity in the dimerization of coumarin 4 (Supplemental Figure S27). ${ }^{38,39}$ To assess the activity and scalability of this enzymatic reaction, analytical scale biotransformations were conducted in Saccharomyces cerevisiae heterologously expressing the gene encoding KtnC. ${ }^{39}$ As previously demonstrated by Müller and coworkers, the formation of the expected dimeric product 7 was observed; however, constraints on substrate loading in S. cerevisiae were not amenable to preparative scale reactions (Supplemental Figure S28). To develop a more scalable wholecell biocatalytic platform in yeast, the gene encoding $\mathrm{KtnC}$ was incorporated into the genome of Pichia pastoris. This new whole-cell system allowed for more than a three-fold increase in the total formation of biaryl product, enabling preparative scale reactions (see Supplemental Section VI). ${ }^{40}$

With the optimized biocatalytic platform in hand, we sought to move beyond natural dimerization reactions to unnatural cross-coupling chemistry. We hypothesized that the binding of two non-equivalent phenolic substrates in the enzyme active site would overcome traditional stereoelectronic limitations in small molecule-mediated oxidative cross-coupling reactions. In a proof-of-concept experiment, coumarins $\mathbf{4}$ and $\mathbf{1 6}$ were both added to a culture producing $\mathrm{KtnC}$, leading to the formation of unnatural cross-coupled products. Further experimentation demonstrated that tuning the stoichiometry of the cross-coupling partners could maximize cross-coupled product formation over dimerization (Supplemental Figure S30). Using this strategy for KtnC-catalyzed cross-coupling reactions, substrate promiscuity was observed with a panel of coumarin substrates (Figure 2a). Initial cross-coupling reactions between 4 and 9-12 demonstrated that maintaining similar substitution patterns to 4 at the $\mathrm{C} 4$ and $\mathrm{C} 5$ positions is beneficial but not required for activity. Additionally, $\mathrm{KtnC}$ tolerated a range of coumarins bearing electron-rich and electron-deficient substituents (13-16), diverging from the stereoelectronic restrictions that typically govern small molecule-catalyzed oxidative cross-coupling reactions. ${ }^{16,17}$ Added steric bulk at the $\mathrm{C} 4$ and C5 positions of coumarins 17-22 was also readily accepted. In contrast to the high levels of reactivity achieved in coumarin cross-coupling reactions with $\mathrm{KtnC}$, modest to no cross-coupling reactivity was observed for these substrate pairs when subjected to established methods for the oxidative coupling of phenolic substrates (Supplemental Table S1). ${ }^{13,21,41-43}$

The majority of the cross-coupling reactions examined maintained the site- and atroposelectivity exhibited in the natural dimerization reaction. For example, the major product resulting from the crosscoupling between 4 and 18 harbored the 8,8'-connectivity and was formed with excellent atroposelectivity (see 23). However, in addition to the expected 8,8'-product, a minor cross-coupled product was observed in reactions with 4 and coumarins bearing increased steric bulk at the C4 position (18-22; Figure 2b). Further characterization of this product revealed a rare 6,6'-connectivity, with cross-coupling to the butyl ester (22) completely shifting the preferencefrom the formation of the 8,8'-product (25) to the 6,6'-product (26) in a 2:5 ratio. Notably, both prefunctionalization and direct oxidative coupling strategies were unsuccessful in generating the 6,6'-isomer in these cross-coupling reactions, highlighting the stereoelectronic biases that impede access to specific connectivities using small molecule-based strategies (Supplemental Figure S8 and S10). ${ }^{16,17}$

In addition to forming a range of unsymmetrical bicoumarin products, a panel of more diverse phenolic substrates were tested as cross-coupling partners for coumarin 4 (Figure 2c). In general, superior reactivity was observed with phenolic substrates that resembled native substrate 4; however, as the substrate structures diverged further from the native coumarin scaffold, significant decreases in the crosscoupling activity and site-selectivity occurred. We hypothesized that the limitations in substrate scope and chemo-, site-, and atroposelectivity could be overcome with protein engineering.

We anticipated that the successful engineering of a cross-coupling catalyst ( $\mathrm{LxC}$ ) could transform a low-yielding, unselective biocatalytic reaction into a practical method for synthesis. The cross-coupling between coumarin $\mathbf{1 0}$ and 2-naphthol (31) was selected as the target reaction due to the low levels of 


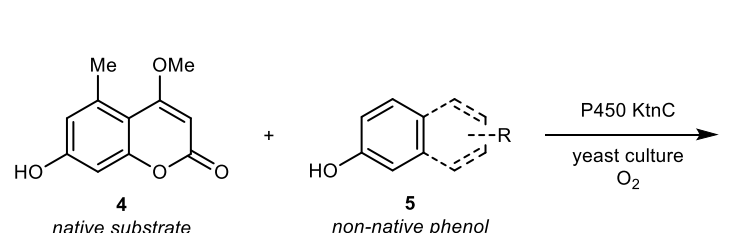

a Cross-coupling reactions with coumarins

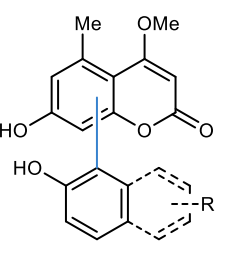

cross-coupled product

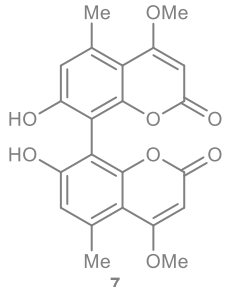

ative dimer

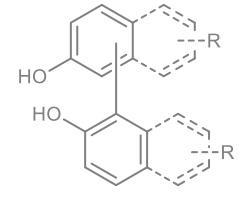<smiles>COc1cc(=O)oc2cc(O)ccc12</smiles><smiles>CCOc1cc(=O)oc2cc(O)c(C)c(OCC)c12</smiles>

b Site- and atroposelectivity trends<smiles>COc1cc(=O)oc2c(-c3c(O)cc(C)c4c(C)cc(O)c(O)c34)c(O)cc(C)c12</smiles>

$95 \%{ }^{\mathrm{a}}$, $>99: 1 \mathrm{er}$

native dimer $\left(8,8^{\prime}\right)$

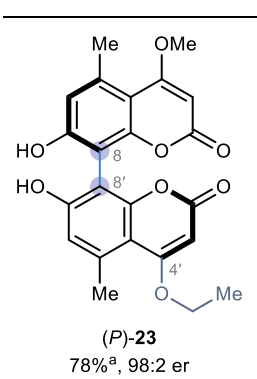

major product $(8,8)$

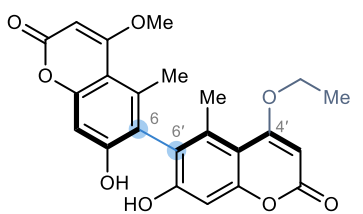

(P)-24

$10 \%{ }^{\mathrm{a}}, 79: 21 \mathrm{er}$

minor product $(6,6)$

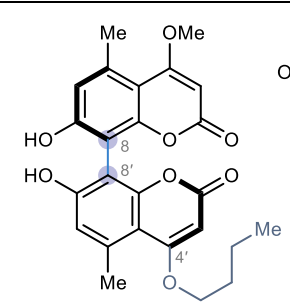

$(P)-25$

$5 \%$ b $, 94: 6$ er

minor product $\left(8,8^{\prime}\right)$

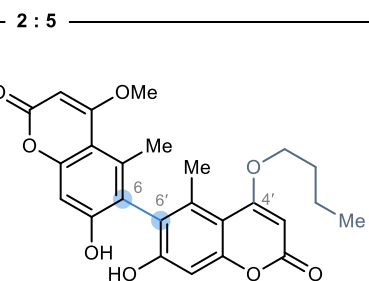

26

$12 \%$ b $, 50: 50$ er

major product $\left(6,6^{\prime}\right)$

C Cross-coupling reactions with diverse phenolic substrates

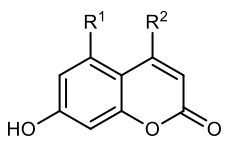

9-22, up to $87 \%$

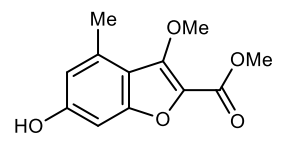

27, $52 \%$ ( $44 \%$ dimer)

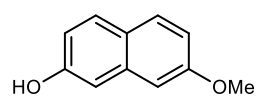

$28,20 \%$<smiles>Oc1ccc2c(c1)[nH]c1ccccc12</smiles>

29, $15 \%$ ( $82 \%$ dimer)

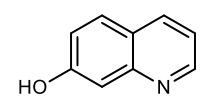

30, $14 \%$ (72\% dimer)

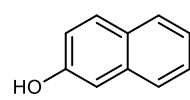

$31,2 \%$

(65\% dimer)

decreasing cross-coupling activity

Fig. 2 | Scope of oxidative cross-coupling reactions catalyzed by wild-type $\mathbf{P 4 5 0}$ KtnC. a, KtnC displayed promiscuity in the crosscoupling of native coumarin 4 with a range of non-equivalent coumarins with varying stereoelectronic properties. b, Most cross-coupling reactions maintained the site- and atroposelectivity displayed by the native product 7, with the exception of the formation of a rare 6,6 ' product (24 and 26). c, KtnC catalyzed the cross-coupling with a diverse set of phenolic substrates, albeit with decreased reactivity and selectivity as the substrate structures deviated further from the native coumarin scaffold. Reaction conditions: $P$. pastoris cells expressing $\mathrm{KtnC}$ in the presence of 4 and 5 in a 1:10 molar ratio with $25-100 \mu \mathrm{M} 4$ or 1:1 molar ratio, $100 \mu \mathrm{M} \mathrm{(25-26)}$ at $30{ }^{\circ} \mathrm{C}$ with shaking at $235 \mathrm{rpm}$ for 48-72 h; cross-coupling reactions reported as percent conversions, ${ }^{a}$ percent yields, or ${ }^{\mathrm{b}}$ relative percent yields; competing dimerization reported as percent conversions in paranthesis.

reactivity and site-selectivity ( $<3 \%$ combined yield of four cross-coupled products) displayed by wildtype KtnC (Figure 3a and Supplemental Figure S66). Protein libraries were generated through semirational mutagenesis, resulting in thousands of variants with one or multiple substitutions within $12 \AA$ of the active site. Variants were screened in high-throughput yeast biotransformations and analyzed for total cross-coupled product formation by RapidFire mass spectrometry (MS). Reaction mixtures containing 
higher levels of the cross-coupled product were then analyzed for cross-coupling site-selectivity in a second-tier screen using LC-MS (Figure 3b). Ultimately, this two-tier MS screen enabled rapid identification of variants with improved activity and site-selectivity for the targeted cross-coupling reaction.

Through iterative rounds of directed evolution, the total activity and site-selectivity in the desired cross-coupling reaction was progressively improved (Figure 3c). Initially, improvements in total activity were targeted, resulting in a 19-fold increase in total cross-coupled product formation over the first two rounds of engineering, while keeping competing levels of coumarin dimerization low. However, the siteselectivity of the reaction was undesirable, with the $\mathrm{C}-\mathrm{O}$ cross-coupled product $(\mathbf{3 5})$ representing nearly half of all the cross-coupled products formed by LxC2. Therefore, variants with improved site-selectivity were targeted in subsequent engineering rounds, leading to greater site-selectivity for the desired 8, $1^{\prime}$ product (32). Ultimately, a biocatalyst with a 92-fold improvement in activity was engineered through five rounds of evolution to form cross-coupled product 32. Preparative scale biotransformations with LxC5 resulted in the isolation of cross-coupled product 32 in a 50\% yield (Supplemental Section VI). The efficiency of this reaction was further exemplified in comparison to tradition synthetic approaches toward 32, with a direct oxidative cross-coupling using a copper cataly st giving a 19\% yield (Supplemental Table S1, Entry $2 \mathrm{a})^{41}$ and a Suzuki cross-coupling strategy providing a $26 \%$ yield over four steps (Supplemental Scheme S2).

As variants were engineered selecting for increased cross-coupling yield and site-selectivity, a decrease in the atroposelectivity of the reaction was observed from an 80:20 er with the wild-type enzyme to a 52:48 er with LxC5 (Supplemental Figure S72). To regain cataly st control over atroposelectivity, two additional rounds of engineering were performed using a combination of RapidFire and chiral LC-MS (Figure 3d). A variant (LxC7) that catalyzed the cross-coupling to 32 in a 77:23 er was identified, albeit with a reduction in yield. These results demonstrate that this biocatalytic platform is highly engineerable for the desired activity, site-selectivity, and atroposelectivity, thereby providing a programmable platform for catalyst-controlled oxidative cross-coupling reactions.

In parallel to employing a protein engineering strategy, we hypothesized that alternative wild-type enzymes could provide complementary catalysts for oxidativecross-coupling reactions. A bioinformaticsguided approach was taken by harnessing SSNs as a tool to visualize the natural sequence space surrounding P450s known to mediate intermolecular oxidative dimerization reactions. An SSN was constructed with the entire P450 protein family (Supplemental Figure S26) and then truncated to edges possessing an alignment score greater than 110 with P450s known to dimerize phenolic substrates (Figure 1d). ${ }^{44-47}$ Characterized P450s that clustered together (indicating high levels of sequence similarity) dimerized similar natural substrates; in contrast, characterized enzymes that cluster separately typically transformed different substrate scaffolds (Supplemental Figure S27). Based on these trends, the reactivity of a panel of 23 enzymes was screened. This panel included 20 uncharacterized enzymes as well as three characterized P450s known to dimerize substrates with naphthoquinone and naphthalene cores (see 37 and 38) that differed structurally from coumarin 4 (Figure $4 a$ ). ${ }^{48,49}$

We anticipated that these enzymes would productively catalyze oxidativecross-coupling reactions with naphthols (e.g. 31 and 40) to provide a suite of complementary catalysts to KtnC. Artificial fusion enzymes were engineered by tethering the $\mathrm{P} 450_{\mathrm{RhF}}$ reductase domain (RhFRed) to the C-terminus of each $\mathrm{P} 450$ to reconstitute the electron transport required for catalytic activity (Supplemental Figure S29).$^{50} \mathrm{In}$ a series of $E$. coli lysate screens with the P450-RhFRed library (Supplemental Table S3), cross-coupled products formed across a diverse set of phenolic substrates to a naphthol partner with surprising promiscuity (Figure 4b). The sequences catalyzing the formation of the highest levels of cross-coupled product were further characterized using purified enzyme. Whereas the cross-coupling yields varied across 
a Challenges in target reaction
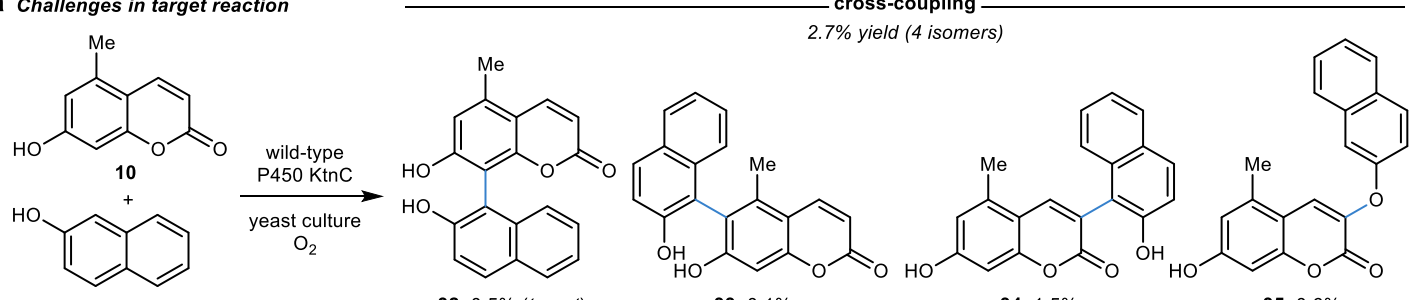

1.7\% yield (4 isomers)

31

$32,0.5 \%$ (target)

33, $0.1 \%$

34, $1.5 \%$

$35,0.6 \%$

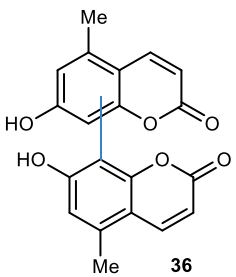

b Strategy for directed evolution

enzyme libraries

variants screened for cross-coupling activity

$34,1.5 \%$

Me $\quad 36$
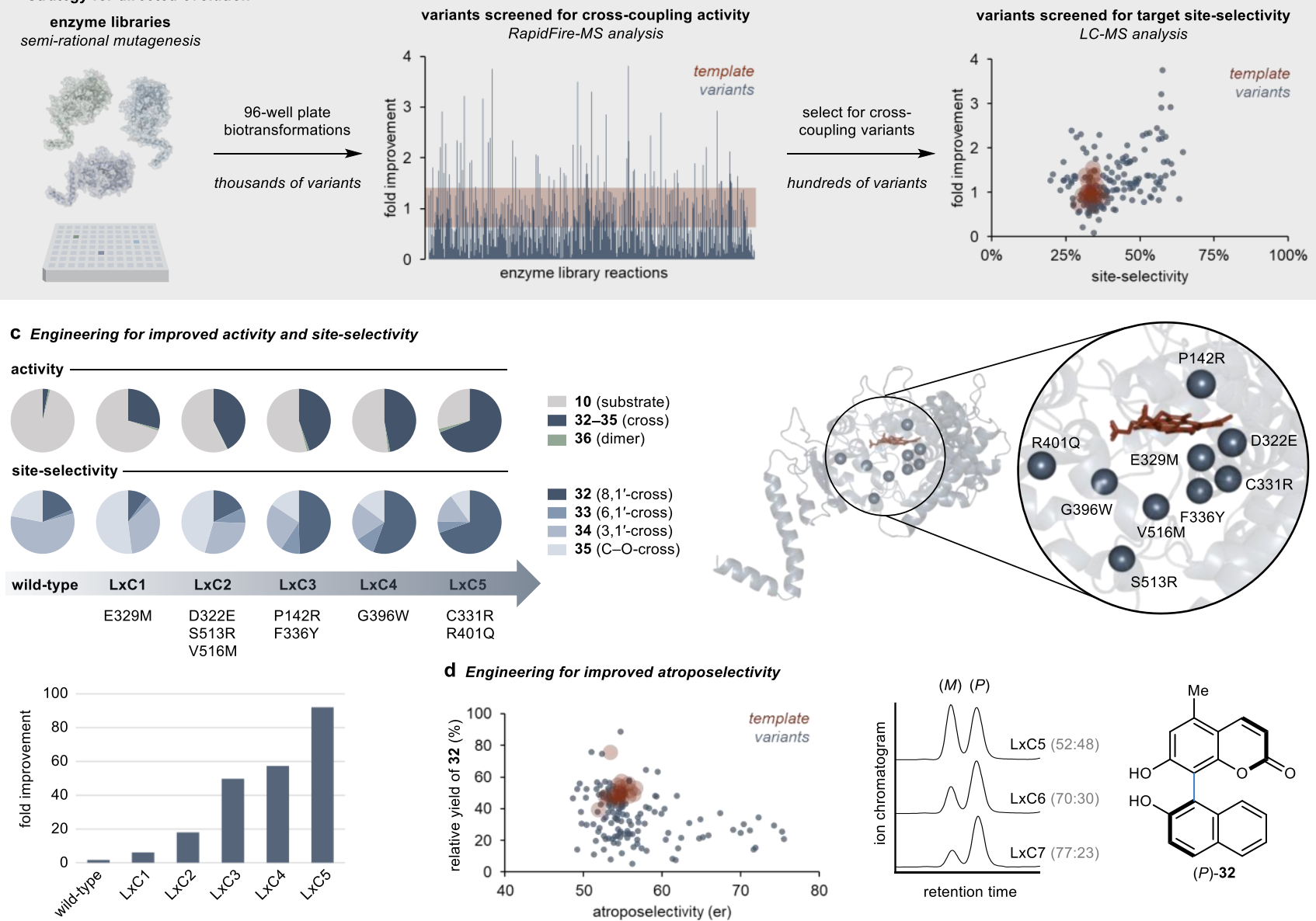

Fig. 3 | Engineering P450 biocatalysts for improved activity and selectivity. a, Target reaction selected for directed evolution campaign, with wild-type $\mathrm{KtnC}$ catalyzing the cross-coupling at a $2.7 \%$ yield to four different cross-coupled isomers, in addition to dimeric products. $\mathbf{b}$, Strategy for the directed evolution of P450s for improved activity and site-selectivity employing semi-rational mutagenesis and a two-tier mass spectrometry screen, as represented by data from round three of evolution above. c, Over five rounds of evolution, a total of nine activesite mutations were incorporated to provide $\mathrm{LxC} 5$ with 92 -fold improvement in activity and site-selectivity toward the target cross-coupled product 32. d, In addition to site-selectivity, the atroposelectivity of the reaction was demonstrated to be tunable with active site mutations.

the diverse set of reactions, the transformations favored the formation of a major cross-coupled product (see 43-46) over competing dimeric product formation (Supplemental Figures S73-S82), demonstrating both chemo- and site-selectivity. Moreover, two of the outstanding hits were novel sequences with previously uncharacterized function (A0A1J4PSC8 and A0A6B3DVM9). Altogether, these results demonstrate the complementary reactivity achievable with different $\mathrm{P} 450$ catalysts and provide biocatalytic access to diverse biaryl scaffolds .

The challenge of overriding innate reactivity and selectivity in oxidative coupling reactions has limited the reliable use of this method for convergent synthetic routes. The presented biocatalytic method 
overcomes reactivity hurdles by surpassing stereoelectronic limitations and redefining substrates that can be efficiently cross-coupled. Furthermore, using a large molecule ligand provides the opportunity to achieve catalyst-controlled site- and atroposelectivity in biaryl coupling reactions. The wealth of natural catalysts available, along with the demonstrated ability to tune enzymes for the desired substrate scope, reactivity, and selectivity, positions this biocatalytic approach to be a powerful tool in the convergent assembly of biaryl molecules.

a strategy for identification of new catalysts
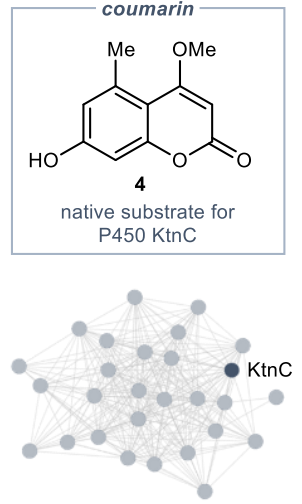

b Cross-coupling reactions with bacterial P450s<smiles>Oc1ccc2ccc(O)cc2c1</smiles>

5<smiles>Cc1cc(O)c(-c2c(O)ccc3ccccc23)c2oc(=O)ccc12</smiles>

32

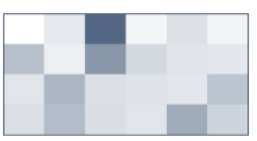

CYP158A2: $7 \%(57: 43)$ KtnC: $0.5 \%(19: 81)$
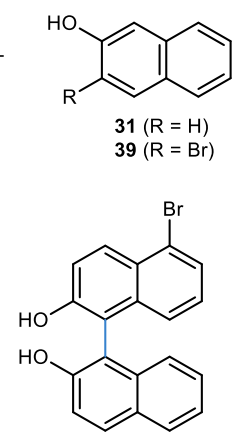

43

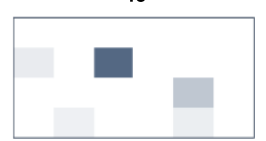

CYP158A2: $13 \%$ yield $(94: 6)$

KtnC: no reaction

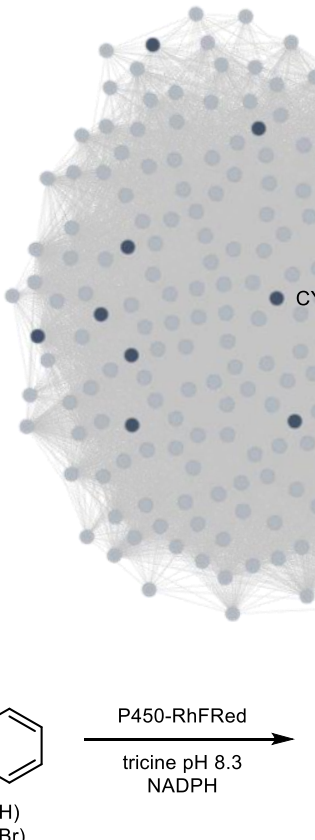

Fig. 4 | Exploring alternative sequence space for catalysts with complementary reactivity. a, Strategy for selection of alternative catalysts for cross-coupling reactions using SSNs. b, Bacterial fusion enzymes (P450-RhFRed) catalyze cross-coupling reactions with naphthols, demonstrating complementary reactivity to $\mathrm{KtnC}$. The top hits from an initial screen of 23 enzymes were purified for further quantification of reaction yield and selectivity. Reaction conditions: $10 \mu \mathrm{M}$ purified P450-RhFRed in the presence of $5 \mathrm{mM}$ glucose-6-phosphate, $1 \mathrm{mM}$ $\mathrm{NADP}^{+}, 1 \mathrm{U} / \mathrm{mL}$ glucose-6-phosphate dehydrogenase, and substrates $(1 \mathrm{mM} 39$ and $1 \mathrm{mM} 31$ or 40 in lysate reactions; $100 \mu \mathrm{M} 39$ and 300 $\mu \mathrm{M} 31$ or 40 in reactions with purified enzyme) in tricine buffer $\mathrm{pH} 8.3$ at $30{ }^{\circ} \mathrm{C}$ for $16 \mathrm{~h}$; cross-coupling reactions reported as percent yields to major product with cross-coupling site-selectivity in paranthesis. 
Online content Any methods, additional references, Nature Research reporting summaries, source data, extended data, supplementary information, acknowledgements, peer review information; details of author contributions and competing interests; and statements of data and code availability are available at https://doi.org/xxxx.

\section{References}

1. Yet, L. Privileged Structures in Drug Discovery: Medicinal Chemistry and Synthesis . First edn, 83-154 (John Wiley \& Sons, Inc., 2018).

2. Yoon, T. P. \& Jacobsen, E. N. Privileged chiral catalysts. Science 299, 1691-1693 (2003).

3. Yin \& Liebscher, J. Carbon-carbon coupling reactions catalyzed by heterogeneous palladium catalysts. Chem. Rev. 107, 133-173 (2007).

4. Boström, J., Brown, D. G., Young, R. J. \& Keserü, G. M. Expanding the medicinal chemistry synthetic toolbox. Nat. Rev. Drug Discov. 17, 709-727 (2018).

5. Roughley, S. D. \& Jordan, A. M. The medicinal chemist's toolbox: an analysis of reactions used in the pursuit of drug candidates. J. Med. Chem. 54, 3451-3479 (2011).

6. Yin, J., Rainka, M. P., Zhang, X.-X. \& Buchwald, S. L. A highly active Suzuki cataly st for the synthesis of sterically hindered biaryls: novel ligand coordination. J. Am. Chem. Soc. 124, 11621163 (2002).

7. Cammidge, A. N. \& Crépy, K. V. L. Synthesis of chiral binaphthalenes using the asymmetric Suzuki reaction. Tetrahedron 60, 4377-4386 (2004).

8. Martin, R. \& Buchwald, S. L. Palladium-catalyzed Suzuki-Miyaura cross-coupling reactions employing dialkylbiaryl phosphine ligands. Acc. Chem. Res. 41, 1461-1473 (2008).

9. Valente, C. et al. The development of bulky palladium NHC complexes for the most-challenging cross-coupling reactions. Angew. Chem. Int. Ed. 51, 3314-3332 (2012).

10. Patel, N. D. et al. Computationally assisted mechanistic investigation and development of Pdcatalyzed asymmetric Suzuki-Miyaura and Negishi cross-coupling reactions for tetra-orthosubstituted biaryl synthesis. ACS Catal. 8, 10190-10209 (2018).

11. Ackermann, L., Potukuchi, H. K., Althammer, A., Born, R. \& Mayer, P. Tetra-ortho-substituted biaryls through palladium-catalyzed Suzuki-Miyaura couplings with a diaminochlorophosphine ligand. Org. Lett. 12, 1004-1007 (2010).

12. Brown, D. G. \& Boström, J. Analysis of past and present synthetic methodologies on medicinal chemistry: where have all the new reactions gone? J. Med. Chem. 59, 4443-4458 (2016).

13. Yang, Y., Lan, J. \& You, J. Oxidative C-H/C-H coupling reactions between two (hetero)arenes. Chem. Rev. 117, 8787-8863 (2017).

14. Kozlowski, M. C. Oxidative coupling in complexity building transforms. Acc. Chem. Res. 50, 638643 (2017).

15. Lee, Y. E., Cao, T., Torruellas, C. \& Kozlowski, M. C. Selective oxidative homo- and crosscoupling of phenols with aerobic catalysts. J. AM. Chem. Soc. 136, 6782-6785 (2014).

16. Nieves-Quinones, Y. et al. Chromium-salen catalyzed cross-coupling of phenols: mechanism and origin of the selectivity. J. Am. Chem. Soc. 141, 10016-10032(2019).

17. Shalit, H., Dyadyuk, A. \& Pappo, D. Selective oxidative phenol coupling by iron catalysis. J. Org. Chem. 84, 1677-1686 (2019).

18. Reiss, H. et al. Cobalt(II)[salen]-catalyzed selective aerobic oxidative cross-coupling between electron-rich phenols and 2-naphthols. J. Org. Chem. 84, 7950-7960 (2019). 
19. Röckl, J. L., Schollmeyer, D., Franke, R. \& Waldvogel, S. R. Dehydrogenative anodic C-C coupling of phenols bearing electron-withdrawing groups. Angew. Chem. Int. Ed. 59, 315-319 (2020).

20. Kang, H. et al. Enantioselective vanadium-catalyzed oxidative coupling: development and mechanistic insights. J. Org. Chem. 83, 14362-14384 (2018).

21. Libman, A. et al. Synthetic and predictive approach to unsymmetrical biphenols by iron-catalyzed chelated radical-anion oxidative coupling. J. Am. Chem. Soc. 137, 11453-11460 (2015).

22. Morimoto, K., Sakamoto, K., Ohshika, T., Dohi, T. \& Kita, Y. Organo-iodine(III)-catalyzed oxidative phenol-arene and phenol-phenol cross-coupling reaction. Angew. Chem. Int. Ed. 55, 3652-3656 (2016).

23. More, N. Y. \& Jeganmohan, M. Oxidative cross-coupling of two different phenols: an efficient route to unsymmetrical biphenols. Org. Lett. 17, 3042-3045 (2015).

24. Egami, H. \& Katsuki, T. Iron-catalyzed asymmetric aerobic oxidation: oxidative coupling of 2 naphthols. J. Am. Chem. Soc. 131, 6082-6083 (2009).

25. Hovorka, M., Günterova, J. \& Zavada, J. Highly selective oxidative cross-coupling of substituted 2-naphthols: a convenient approach to unsymmetrical 1, 1'-binaphthalene-2, 2'-diols. Tet. Lett. 31, 413-416 (1990).

26. Li, X., Hewgley, J. B., Mulrooney, C. A., Yang, J. \& Kozlowski, M. C. Enantioselective oxidative biaryl coupling reactions catalyzed by 1,5-diazadecalin metal complexes: efficient formation of chiral functionalized BINOL derivatives. J. Org. Chem. 68, 5500-5511 (2003).

27. Tian, J.-M. et al. Copper-complex-catalyzed asymmetric aerobic oxidative cross-coupling of 2naphthols: enantioselective synthesis of 3,3'-substituted C1-symmetric BINOLs. Angew. Chem. Int. Ed. 58, 11023-11027 (2019).

28. Bringmann, G. et al. Atroposelective synthesis of axially chiral biaryl compounds. Angew. Chem. Int. Ed. 44, 5384-5427 (2005).

29. Kočovský, P., Vyskočil, Š. \& Smrčina, M. Non-symmetrically substituted 1,1'-binaphthyls in enantioselective catalysis. Chem. Rev. 103, 3213-3246 (2003).

30. Kozlowski, M. C., Morgan, B. J. \& Linton, E. C. Total synthesis of chiral biaryl natural products by asymmetric biaryl coupling. Chem. Soc. Rev. 38, 3193-3207 (2009).

31. Bringmann, G., Gulder, T., Gulder, T. A. M. \& Breuning, M. Atroposelective total synthesis of axially chiral biaryl natural products. Chem. Rev. 111,563-639(2011).

32. Aldemir, H., Richarz, R. \& Gulder, T. A. The biocataly tic repertoire of natural biaryl formation. Angew. Chem. Int. Ed. 53, 8286-8293 (2014).

33. Mate, D. M. \& Alcalde, M. Laccase: a multi-purpose biocataly st at the forefront of biotechnology. Microb. Biotechnol. 10, 1457-1467 (2017).

34. Sagui, F. et al. Laccase-catalyzed coupling of catharanthine and vindoline: an efficient approach to the bisindole alkaloid anhydrovinblastine. Tetrahedron 65, 312-317 (2009).

35. Obermaier, S., Thiele, W., Fürtges, L. \& Müller, M. Enantioselective phenol coupling by laccases in the biosynthesis of fungal dimeric naphthopyrones. Angew. Chem. Int. Ed. 58, 9125-9128 (2019).

36. Fasan, R. Tuning P450 enzymes as oxidation catalysts. ACS Catal. 2, 647-666 (2012).

37. Liu, J., Liu, A. \& Hu, Y. Enzymatic dimerization in the biosynthetic path way of microbial natural products. Nat. Prod. Rep. 38, 1469-1505 (2021).

38. Gil Girol, C. et al. Regio-and stereoselective oxidative phenol coupling in Aspergillus niger. Angew. Chem. Int. Ed. 51, 9788-9791 (2012). 
39. Mazzaferro, L. S., Huttel, W., Fries, A. \& Müller, M. Cytochrome P450-catalyzed regio- and stereoselective phenol coupling of fungal natural products. J. Am. Chem. Soc. 137, 12289-12295 (2015).

40. Chakrabarty, S., Wang, Y., Perkins, J. C. \& Narayan, A. R. H. Scalable biocatalytic C-H oxyfunctionalization reactions. Chem. Soc. Rev. 49, 8137-8155 (2020).

41. Noji, M., Nakajima, M. \& Koga, K. A new catalytic system for aerobic oxidative coupling of 2naphthol derivatives by the use of $\mathrm{CuCl}$-amine complex: a practical synthesis of binaphthol derivatives. Tet. Lett. 35, 7983-7984 (1994).

42. Nakajima, M. Synthesis and application of novel biaryl compounds with axial chirality as catalysts in enantioselective reactions. Yakugaku Zasshi 120, 68-75 (2000).

43. Langeslay, R. R. et al. Catalytic applications of vanadium: a mechanistic perspective. Chem. Rev. 119, 2128-2191 (2018).

44. Shannon, P. et al. Cytoscape: A software environment for integrated models of biomolecular interaction networks. Genome Res. 13, 2498-2504 (2003).

45. Gerlt, J. A. et al. Enzyme Function Initiative-Enzyme Similarity Tool (EFI-EST): a web tool for generating protein sequence similarity networks. Biochim. Biophys. Acta. 1854, 1019-1037 (2015).

46. Zallot, R., Oberg, N. O. \& Gerlt, J. A. 'Democratized' genomic enzymology web tools for functional assignment. Curr. Opin. Chem. Biol. 47, 77-85 (2018).

47. Zallot, R., Oberg, N. \& Gerlt, J. A. The EFI web resource for genomic enzymology tools: leveraging protein, genome, and metagenome databases to discover novel enzymes and metabolic pathways. Biochemistry 58, 4169-4182 (2019).

48. Funa, N., Funabashi, M., Ohnishi, Y. \& Horinouchi, S. Biosynthesis of hexahydroxyperylenequinone melanin via oxidative aryl coupling by cytochrome P-450 in Streptomyces griseus. J. Bacteriol. 187, 8149-8155 (2005).

49. Zhao, B. et al. Binding of two flaviolin substrate molecules, oxidative coupling, and crystal structure of Streptomyces coelicolor A3(2) cy tochrome P450 158A2. J. Biol. Chem. 280, 1159911607 (2005).

50. Li, S., Podust, L. M. \& Sherman, D. H. Engineering and analysis of a self -sufficient biosynthetic cytochrome P450 PikC fused to the RhFRED reductase domain. J. Am. Chem. Soc. 129, 1294012941 (2007).

Data availability All data are available from the corresponding author upon reasonable request.

Acknowledgements This research was supported by funds from the University of Michigan's Life Sciences Institute and the Chemistry Department, Alfred P. Sloan Foundation, and Research Corporation Cottrell Scholars program. Initial studies on the selectivity of dimerization reactions were supported by the NIH R35 GM124880, and protein engineering (rounds 4-7) and bioinformatic-based library generation were supported by generous funds from the Novartis Global Scholars Program. L. E. Z. is grateful to the NIH National Center for Complementary \& Integrative Health of the NIH F31 AT010973, J. A. Y. thanks the National Science Foundation GRFP, A. L. L. acknowledges the Rackham Graduate School (UM) and the NIH F31 NS1 11906 for funding. We thank M. Müller for supplying the expression vector containing KtnC and D. Sherman for supplying the expression vector containing RhFRed. We thank E. A. Meucci, E. C. Bornowski, and J. B. Pyser for assistance with the synthesis of substrates and C-H. Chiang for help with $\mathrm{CD}$ acquisition. 
Author Contributions L. E. Z., J. A. Y., S. C., M. E. H., and A. R. H. N. designed experiments and wrote the manuscript with feedback from all authors. L. E. Z. and A. L. L. generated yeast strains used in this work. L. E. Z. performed protein engineering and generated sequence similarity networks presented in this work. L. E. Z., J. A. Y., M. E. H., and L. A. M. M. conducted biocatalytic reactions. J. A. Y., S. C., and M. E. H. synthesized substrates and racemic product standards. S. C. carried out product isolation from preparative scale biocatalytic reactions. L. A. J. calculated CD spectra and assigned the absolute configuration of products.

Competing Interests The authors have no conflicts of interest to report.

\section{Additional information}

Supplementary information The online version contains supplementary material available at https://doi.org/Xxxx.

Correspondence and requests for materials should be addressed to A. R. H. N.

Peer review information Nature thanks the anonymous reviewers for their contribution to the peer review of this work. 